Bull. Fac. Agric., Cairo Univ., 63: 386-395 (2012).

\title{
MULTIPLE REGRESSION AND CORRELATION ANALYSIS OF EARLINESS AND POTENTIAL PRODUCTION TRAITS OF FIVE COTTON VARIETIES (Gossypium barbadense L.) GROWN UNDER DIFFERENT ENVIRONMENTS
}

(Received:27.9.2012)

\author{
By \\ F. M. Ismail, M .D .H. Dewdar and A. H. A. Mahdi \\ Agronomy Department, Faculty of Agriculture, Fayoum University, Egypt
}

\begin{abstract}
The present experiments were carried out at the Farm of the Faculty of Agriculture, El Fayoum University, in the summer seasons of 2010 and 2011 in a split plot design with five replicates, to investigate the effects of different sowing times, as an indication of different climatic environments i.e. micro-environments on earliness, yield and its components in some Egyptian genotypes.

The obtained results indicated that the studied plant characters were significantly affected by microenvironments. However, its effect was not observed in some earliness measurements i.e. node number of the first sympodium, the period between the first bud and the first flower appearance, the period between the first flower appearance and the first boll opening and mean maturity date. Early sowing recorded the highest values of the studied characters. While, delayed sowing to the $1^{\text {st }}$ of April significantly decreased the values of the characters. Significant differences among varieties were found in all the studied traits over the three environments except the period among the first bud initiation and the first flower appearance and the period between the first flower appearance and the first boll opening. Giza 90 gave the highest seed and lint cotton yields. The results obtained clearly indicated that the treatment combination comprising early time on the first of March with the variety Giza 90 proved to gave the highest values of seed and lint cotton yields.

It could be concluded that earliness traits were able to result in a linear regression model for all the five tested genotypes. At least two of these traits were included in the yield per feddan model of the five varieties. Consequently, this way of estimating earliness could be favored over the other methods used regardless of the variety studied.
\end{abstract}

Key words: correlation analysis, cotton genotypes, micro-environments, multiple regression, production traits.

\section{INTRODUCTION}

Earliness of maturity in cotton is a complex trait, influenced by a number of morphological and phonological aspects besides the environmental conditions. The microclimatic conditions prevailing during the growth period and maturity might affect the performance of cotton plants throughout the phonological stages of growth and yield and its components. The progress of a plant from germination to maturity depends on the interplay of genetic and environmental factors which determine the timing and rate of developmental processes. For the implementation of the microclimatic changes they must be quantified.

The correlation coefficient analysis helps to determine the nature and magnitude of the relationship between any two characters. But it does not consider dependence of one variable over the other. Further more, the direct contribution of each component cannot be differentiated by simple correlations. Richmoned and Ray (1966), Ray and Richmoned (1966) and Abdel-Rahman (1983), reported significant positive correlations between some phonological traits i.e. first flower, boll opening, sympodial branch, node number and the total yield in cotton. Negative correlations between the first fruiting node, the first effective boll and days to first flower has been found by Gopang (2003).

In this respect, El-Shaer et al. (1984), Seyam et al. (1984), Ismail and Al-Enani (1986) and ElBeily et al. (1996) used the technique of stepwise multiple regression and correlation analysis for 
assessing the relationships between yield and its components.

Moreover, Badr et al. (2001), Hassan and Abdel-Aziz (2004) and Saeed et al. (2008) on Egyptian cotton found significant correlation among earliness measurements i.e. position of the first fruiting node, days to first boll opening, mean maturity date, production rate index and seed cotton yield. While, Muhammad et al. (2003 and 2006), Karademir et al. (2009), Kausar et al. (2005), Shazia et al. (2010) and Kazerani (2012) on Gossypium hirsutum L., showed that the node of the first fruiting branch, monopodial branches per plant, the number of open bolls per plant and boll weight were positive and significantly correlated with yield.

The present study used regression analysis and correlation calculation for the information of interrelationships between earliness and cotton yield traits to determine the traits accounted for most of the variation in yield under different environmental conditions.

\section{MATERIAL AND METHODS}

The present study was carried out at the experimental farm of the Faculty of Agriculture, El-Fayoum University during the two growing seasons of 2010 and 2011. Three sowing dates as indication of different micro-environments were used i.e. early time on the first of March, medium time on the second half of March and the late time on the first of April. The expression of sowing times is used to represent specific microenvironment in periods of days instead of sowing time. The fifteen day averages of relative humidity and the maximum and minimum temperature degrees during the two growing seasons are reported in (Table 1). In order to calculate the heat units (H.U.) according to Young et al. (1980) the following equation was used:

H.U. $=$ [mean daily (min. and max.) temperature $-\mathrm{K}\left(\right.$ Zero growth $\left.\left.=12.8{ }^{\circ} \mathrm{C}\right)\right]$.

Five Egyptian cotton varieties (Gossypium barbadense L.), i.e. Giza 70 and Giza 88 classified as extra long staple and Giza 80, Giza 86 and Giza 90 classified as long staple varieties were used. The aforementioned varieties were grown in a split-plot design with five replications where sowing times as micro - environments were allotted in the main plots and varieties were arranged in sub plots. The plot size was $(3 \times 3.5$ $\mathrm{m})=10.5 \mathrm{~m}^{2}$. All other practices were done according to the recommendations.

\section{Recording of observations}

Yield and its components for each entry tested were assigned as $\left(\mathrm{Y}_{\mathrm{i}}\right)$ dependent variable where:

$\mathrm{Y}_{1}=$ Yield $/$ feddan (kantar.).

$\mathrm{Y}_{2}=$ Yield / plant (g.).

$\mathrm{Y}_{3}=$ No. of bolls / plant.

$\mathrm{Y}_{4}=$ Boll weight (g.).

$\mathrm{Y}_{5}=$ Lint percentage $(\%)$.

$\mathrm{Y}_{6}=$ Seed index (g.).

Table (1): Fifteen days average of relative humidity and air temperature at Fayoum region during the two growing seasons. *

\begin{tabular}{|c|c|c|c|c|c|c|c|c|}
\hline \multirow{3}{*}{ Intervals } & \multirow{2}{*}{\multicolumn{2}{|c|}{$\begin{array}{l}\text { Relative } \\
\text { humidity }\end{array}$}} & \multicolumn{6}{|c|}{ Air temperature } \\
\hline & & & \multicolumn{2}{|c|}{ Max. } & \multicolumn{2}{|c|}{ Min. } & \multicolumn{2}{|c|}{ H.U. } \\
\hline & 2010 & 2011 & 2010 & 2011 & 2010 & 2011 & 2010 & 2011 \\
\hline $1 / 2-15 / 2$ & 72 & 73 & 23.9 & 22.9 & 6.6 & 6.5 & 17.7 & 16.6 \\
\hline $16 / 2-28 / 2$ & 76 & 78 & 20.4 & 26.2 & 6.2 & 10.1 & 13.8 & 23.5 \\
\hline $1 / 3-15 / 3$ & 74 & 78 & 24.0 & 31.0 & 8.6 & 12.5 & 19.8 & 30.7 \\
\hline $16 / 3-31 / 3$ & 77 & 79 & 22.4 & 24.2 & 7.3 & 10.4 & 16.9 & 21.8 \\
\hline $1 / 4-15 / 4$ & 76 & 77 & 30.0 & 31.2 & 12.1 & 12.9 & 29.3 & 31.3 \\
\hline $16 / 4-30 / 4$ & 75 & 74 & 31.6 & 32.3 & 12.9 & 15.7 & 31.7 & 35.2 \\
\hline $1 / 5-15 / 5$ & 74 & 75 & 30.4 & 35.4 & 14.7 & 16.4 & 32.3 & 39.0 \\
\hline $16 / 5$ - 31/5 & 76 & 74 & 35.1 & 33.0 & 18.6 & $\mathbf{1 7 . 1}$ & 40.9 & 37.3 \\
\hline $1 / 6-15 / 6$ & 72 & 75 & 37.7 & 37.3 & 20.0 & 20.4 & 44.9 & 44.9 \\
\hline $16 / 6-30 / 6$ & 75 & 75 & 38.7 & 39.4 & 21.0 & 22.3 & 46.9 & 48.9 \\
\hline $1 / 7-15 / 7$ & 75 & 77 & 37.7 & 36.2 & 22.4 & 21.8 & 47.3 & 45.2 \\
\hline $16 / 7-31 / 7$ & 76 & 76 & 39.2 & 36.4 & 22.9 & 22.9 & 49.3 & 46.5 \\
\hline $1 / 8-15 / 8$ & 77 & 75 & 37.5 & 40.5 & 22.3 & 24.3 & 47.0 & 52.0 \\
\hline $16 / 8-31 / 8$ & 77 & 75 & 36.6 & 40.0 & 21.4 & 24.6 & 45.2 & 51.8 \\
\hline $1 / 9-15 / 9$ & 76 & 77 & 36.0 & 36.2 & 21.0 & 22.4 & 44.2 & 41.8 \\
\hline $16 / 9-30 / 9$ & 77 & 75 & 34.4 & 34.2 & 20.0 & 21.4 & 41.6 & 42.8 \\
\hline $1 / 10-15 / 10$ & 75 & 74 & 32.7 & 36.5 & 19.3 & 21.3 & 39.2 & 45.0 \\
\hline $16 / 10-31 / 10$ & 76 & 77 & 30.8 & 35.4 & 17.0 & 21.2 & 35.0 & 43.8 \\
\hline
\end{tabular}

* Meteorology Station of the Agricultural Management in Itsa (Administrative Centre in Fayoum). 
$\mathrm{Y}_{7}=$ Lint index (g.).

$\mathrm{X}_{\mathrm{i}}$ variables were assigned to the ten earliness traits as follows:

$\mathrm{X}_{1}=$ Node number of first sympodium.

$\mathrm{X}_{2}=$ Number of days from planting to first bud initiation.

$X_{3}=$ Number of days from planting to first flower appearance.

$\mathrm{X}_{4}=$ The period between first bud and first flower appearance.

$\mathrm{X}_{5}=$ Number of days from planting to first boll opening.

$\mathrm{X}_{6}=$ The period between first flower appearance and first boll opening.

$\mathrm{X}_{7}=$ Earliness index.

$\mathrm{X}_{8}=$ Mean maturity date. It is the mean weight of seed cotton yield on harvest date of several periodic harvests calculated according to Christidis and Harrison (1955) using the following formula:

$\mathrm{MMD}=\left(\mathrm{W}_{1} \mathrm{H}_{1}+\mathrm{W}_{2} \mathrm{H}_{2}+\right.$ $\left.+\mathrm{W}_{\mathrm{n}} \mathrm{H}_{\mathrm{n}}\right)$

Where: $\mathrm{W}=$ weight of seed cotton yield in grams. $\mathrm{H}=$ number of days from planting to harvest.

$1,2 \ldots \ldots \mathrm{n}=$ consecutive period harvest number $(5$ harvests).

$\mathrm{X}_{9}=$ Production rate index. It was calculated by dividing the total seed cotton yield by mean maturity date value which results in relative production rate (amount per unit time) according to Bilboro and Quisenberry (1973). The general formula for this value would be:

PRI $=\left(\mathrm{W}_{1}+\mathrm{W}_{2}+\ldots . \mathrm{W}_{\mathrm{n}}\right)^{2} /\left({ }_{1} \mathrm{H}_{1}+\mathrm{W}_{2} \mathrm{H}_{2}+\ldots \ldots+\mathrm{W}_{\mathrm{n}} \mathrm{H}_{\mathrm{n}}\right)$

Where: $\mathrm{W}=$ weight of seed cotton yield in grams.

$\mathrm{H}=$ number of days from planting to harvest.

$1,2 \ldots \ldots \mathrm{n}=$ consecutive period harvest number $(5$ harvests).

$\mathrm{X}_{10}=$ Bartlett earliness index. Earliness was measured by adopting Bartlett (1973) as follows:

$$
\frac{\mathrm{P} 1+(\mathrm{P} 1+\mathrm{P} 2)+(\mathrm{P} 1+\mathrm{P} 2+\mathrm{P} 3)+\ldots \ldots \mathrm{Pn})}{\mathrm{N}(\mathrm{P} 1+\mathrm{P} 2+\mathrm{P} 3+\ldots \ldots+\mathrm{Pn})}
$$

Where $\mathrm{P} 1, \mathrm{P} 2$ and $\mathrm{Pn}=$ the weight of seed cotton picked during first, second and $\mathrm{n}^{\text {th }}$ picking and $\mathrm{N}$ is the total number of pickings.

Five pickings were carried out by hand throughout the period from 5 August to 9 September with periodic harvest 15 days.

\section{Statistical analysis}

The data obtained were subjected to statistical analysis according to the procedures outlined by Snedecor and Cochran (1981). After using homogeneity test for error variance by using Bartlett's test, combined analysis was performed (Cochran and Cox 1957). The stepwise multiple regression and correlation analyses were carried out according to the procedures outlined by Draper and Smith (1966) to determine the variable which would account for the most variation in crop yield.

\section{RESULTS AND DISCUSSION 3.1.Varietal performance under different micro-environments}

Different measures of earliness of maturity were investigated .Some measures deal with dates, i.e. dates of the $1^{\text {st }}$ bud initiation, the $1^{\text {st }}$ flower appearance, the $1^{s t}$ open boll. Other group of measurements dealt with earliness of the cotton harvesting either as percentage of the $1^{\text {st }}$ pick to the total harvested cotton, or as per day or per feddan production index.

Tables (2) and (3) clarify differences among varieties in earliness. All measures reflected such differences among the studied genotypes. Earliness index (weight of the $1^{\text {st }}$ pick to the total yield) showed mean values for the character in the studied varieties ranged between 75.14 to $64.24 \%$ in the first micro-environment $\left(\mathrm{M}_{1}\right)$, while the values ranged between 70.08 to $60.48 \%$ in $\left(\mathrm{M}_{2}\right)$ and 64.52 to $58.40 \%$ in $\left(\mathrm{M}_{3}\right)$. Based on this earliness criterion, variety Giza 90 showed high values of this character, indicating that this variety exhibits better earliness. Earliness index showed high values with early sowing time (M1) in the two studied seasons. Results might be due to that (M1) is coincided to better growth of cotton plants.

Production rate index, is a measure of earliness which expresses three components i.e. number of days from planting to harvesting, yield of cotton during such period and earliness if a given variety is subjected to picking on a specific date after enough bolls open in the field of experimentation forming the $1^{\text {st }}$ pick. Another picks to the end of the season will give reliable data. As for the effect of sowing time formed micro-climatic i.e M1, M2 and M3 generally, every trait studied for earliness in cotton has it own nature.

On earliness traits, Table (2) shows highly significant differences in most instances supporting the idea that every trait has its own nature. If maturity is expressed as the number of days to $1^{\text {st }}$ square or bloom or boll, the late sown will consume less days towards development. The 
Table (2): Average values of earliness traits as affected by microenvironment for 5 cotton varieties (combined data).

\begin{tabular}{|c|c|c|c|c|c|c|}
\hline \multirow{2}{*}{$\begin{array}{c}\text { Microenvironments } \\
\text { (M) }\end{array}$} & \multicolumn{5}{|c|}{ Varieties (V) } & \multirow{2}{*}{ Mean } \\
\hline & $\begin{array}{c}\text { Giza } \\
90(\mathrm{~V} 1)\end{array}$ & $\begin{array}{c}\text { Giza } \\
88 \text { (V2) }\end{array}$ & $\begin{array}{c}\text { Giza } \\
88(\text { V3) }\end{array}$ & $\begin{array}{c}\text { Giza } \\
86(\text { V4) }\end{array}$ & $\begin{array}{c}\text { Giza } \\
70 \text { (V5) }\end{array}$ & \\
\hline \multicolumn{7}{|c|}{ Node number of first sympodium } \\
\hline Early time (M1) & 5.97 & 6.76 & 6.64 & 5.69 & 7.79 & 6.57 \\
\hline Moderate time (M2) & 5.18 & 7.14 & 5.69 & 6.25 & 7.04 & 6.26 \\
\hline Late time (M3) & 5.02 & 6.61 & 5.46 & 5.72 & 6.51 & 5.86 \\
\hline Mean & 5.39 & 6.84 & 5.93 & 5.88 & 7.11 & 6.23 \\
\hline LSD 5\% & \multicolumn{2}{|c|}{ M = N.S. } & \multicolumn{2}{|c|}{$\mathrm{V}=\mathbf{1 . 1 1}$} & \multicolumn{2}{|c|}{$M \times V=$ N.S. } \\
\hline \multicolumn{7}{|c|}{ Number of days from planting to first bud initiation } \\
\hline Early time (M1) & 39.91 & 47.05 & 42.36 & 44.42 & 48.59 & 44.46 \\
\hline Moderate time (M2) & 37.82 & 44.47 & 40.48 & 41.55 & 45.91 & 42.04 \\
\hline Late time (M3) & 35.04 & 41.23 & 37.53 & 39.01 & 43.98 & 39.35 \\
\hline Mean & 37.59 & 44.25 & 40.12 & 41.66 & 46.16 & 41.95 \\
\hline LSD 5\% & $\mathbf{M}=$ & & $\mathbf{V}=\mathbf{1}$ & .11 & M x V & $=2.66$ \\
\hline Numb & of days fr & n plantin & o first flo & wer appea & rance & \\
\hline Early time (M1) & 88.35 & 94.84 & 91.10 & 93.02 & 97.17 & 92.89 \\
\hline Moderate time (M2) & 85.38 & 92.38 & 88.62 & 90.96 & 94.49 & 90.36 \\
\hline Late time (M3) & 83.16 & 89.49 & 86.07 & 88.35 & 92.07 & 87.82 \\
\hline Mean & 85.63 & 92.23 & 88.59 & 90.77 & 94.58 & 90.36 \\
\hline LSD 5\% & $\mathbf{M}=1$ & & $V=1$. & & M x V & $=2.77$ \\
\hline Numbe & days betv & n first bu & ad first & flower app & pearance & \\
\hline Early time (M1) & 48.44 & 47.79 & 48.74 & 48.60 & 48.57 & 48.32 \\
\hline Moderate time (M2) & 47.56 & 47.91 & 48.14 & 49.40 & 48.58 & 48.37 \\
\hline Late time (M3) & 48.12 & 47.76 & 48.52 & 49.34 & 48.09 & 48.43 \\
\hline Mean & 48.04 & 47.82 & 48.47 & 49.11 & 48.41 & 48.37 \\
\hline LSD 5\% & $\mathbf{M}=$ & T.S. & $\mathbf{V}=1$ & N.S. & Mx I & N.S. \\
\hline & er of day & rom plan & ng to first & boll openi & & \\
\hline Early time (M1) & 134.15 & 139.47 & 136.25 & 137.84 & 141.21 & 137.78 \\
\hline Moderate time (M2) & 131.60 & 137.14 & 133.13 & 135.92 & 138.57 & 135.27 \\
\hline Late time (M3) & 129.54 & 134.32 & $\mathbf{1 3 0 . 5 7}$ & 132.27 & 136.31 & 132.60 \\
\hline Mean & 131.76 & 136.97 & 133.31 & 135.34 & 138.70 & 135.22 \\
\hline LSD 5\% & $\mathbf{M}=$ & & $\mathbf{V}=\mathbf{1}$ & .15 & $\mathbf{M} \times \mathbf{V}$ & 2.31 \\
\hline & r of day & rom plan & g to first & boll openi & & \\
\hline Early time (M1) & 45.80 & 44.64 & 44.85 & 44.82 & 44.05 & 44.77 \\
\hline Moderate time (M2) & 46.22 & 44.76 & 44.51 & 44.97 & 44.08 & 44.83 \\
\hline Late time (M3) & 46.37 & 44.83 & 44.50 & 43.92 & 44.24 & 44.91 \\
\hline Mean & 64.13 & 44.74 & 44.62 & 44.57 & 44.12 & 44.84 \\
\hline LSD 5\% & $\mathbf{M}=$ & & $\mathbf{V}=\mathbf{N}$. & & $M \times V$ & N.S \\
\hline & & Earline & dex & & & \\
\hline Early time (M1) & 74.57 & 68.17 & 71.93 & 70.03 & 63.01 & 69.55 \\
\hline Moderate time (M2) & 69.48 & 62.37 & 65.47 & 64.09 & 61.53 & 64.59 \\
\hline Late time (M3) & 64.20 & 60.24 & 62.53 & 61.31 & 59.50 & 61.56 \\
\hline Mean & 69.42 & 63.48 & 66.49 & 64.70 & 62.06 & 65.23 \\
\hline & $\mathbf{M}$ & 2.28 & $\mathrm{~V}=\mathbf{2}$. & 24 & Mx V & 2.25 \\
\hline
\end{tabular}


Table (2): Cont.

\begin{tabular}{|c|c|c|c|c|c|c|}
\hline \multicolumn{7}{|c|}{ Mean maturity date } \\
\hline Early time (M1) & 147.59 & 148.94 & 143.10 & 146.96 & 151.16 & 146.31 \\
\hline Moderate time (M2) & 140.98 & 148.11 & 142.44 & 146.19 & 150.58 & 145.15 \\
\hline Late time (M3) & 138.43 & 147.12 & 141.75 & 145.04 & 149.71 & 145.12 \\
\hline Mean & 142.33 & 147.89 & 142.43 & 146.18 & 150.48 & 145.86 \\
\hline \multirow{2}{*}{\multicolumn{7}{|c|}{$M \times V=2.23$}} \\
\hline & & & & & & \\
\hline Early time (M1) & 1.96 & 1.18 & 1.75 & 1.39 & 1.19 & 1.47 \\
\hline Moderate time (M2) & 1.74 & 1.05 & $\mathbf{1 . 5 3}$ & 1.29 & 1.02 & 1.35 \\
\hline Late time (M3) & 1.32 & 0.76 & 1.12 & 0.88 & 0.59 & 0.93 \\
\hline Mean & 1.67 & 1.00 & 1.47 & 1.19 & 0.93 & 1.25 \\
\hline LSD $5 \%$ & $\mathbf{M}=$ & & $V=1$ & & $\mathbf{M} \mathbf{x}$ & 0.21 \\
\hline \multicolumn{7}{|c|}{ Bartlett } \\
\hline Early time (M1) & 0.72 & 0.65 & 0.70 & 0.69 & 0.62 & 0.68 \\
\hline Moderate time (M2) & 0.68 & 0.63 & 0.66 & 0.65 & 0.59 & 0.64 \\
\hline Late time (M3) & 0.64 & 0.59 & 0.62 & 0.61 & 0.55 & 0.60 \\
\hline Mean & 0.68 & 0.62 & 0.66 & 0.65 & 0.59 & 0.64 \\
\hline LSD $5 \%$ & \multicolumn{2}{|c|}{$\mathbf{M}=\mathbf{0 . 0 7}$} & \multicolumn{2}{|c|}{$V=0.08$} & $M \times V$ & .12 \\
\hline
\end{tabular}

interaction of sowing time $\mathrm{x}$ variety showed highly significant influences on most earliness measures.

\subsection{Interrelationship among variables of earliness and yield}

The model contained only those variables $\left(\mathrm{x}_{\mathrm{i}}\right)$ which significantly affected the dependent variables y was reported. The model should has significant $(\mathrm{F})$ value for the component due to regression and has the most effective coefficient of determination $\left(\mathrm{R}^{2}\right)$. Those variables which did not add sizable contribution to $\left(\mathrm{R}^{2}\right)$ were not included.

Data in Table (4) indicated that variety Giza 90 , only yield /feddan trait $\left(\mathrm{Y}_{1}\right)$ and no. of open bolls / plant $\left(\mathrm{Y}_{3}\right)$ resulted in model with high $\mathrm{R}^{2}$ value 99.05 and $82.73 \%$, respectively. Other models has less $\mathrm{R}^{2}$ value with a minimum of $49.25 \%$ for lint percentage trait $\left(\mathrm{Y}_{5}\right)$. These results could be explained that the earliness traits did not play a major role in the traits with low value of $\mathrm{R}^{2}$, or that this $\mathrm{X}_{\mathrm{i}}$ did not fit the linear model assumption.

For yield/feddan, the aforementioned model included $X_{7}, X_{2}$ and $X_{1}$. Both $X_{7}$ and $X_{2}$ had positive regression coefficient. Ninety nine percent of the variation in yield per feddan rudely explained by the given linear regression model as indicated by the coefficient of determination $\left(\mathrm{R}^{2}\right.$ $\%)$. In addition, the multiple correlation coefficient value $(\mathrm{R})$ was 0.991 . Consequently, the $\left(\mathrm{X}_{\mathrm{i}}\right)$ trait included in that model contained appropriate biological entities.
No. of open bolls/plant mathematical model included $\mathrm{X}_{7}, \mathrm{X}_{3}, \mathrm{X}_{8}$ and $\mathrm{X}_{4}$. All partial regression coefficients in this model were positive expect $\mathrm{X}_{8}$ which was negative. Approximately eighty three percent of the total variation in No. of open bolls/ plant rudely explained by the given mathematical model as indicated by the coefficient of determination $\left(\mathrm{R}^{2} \%\right)$.

The five other linear regression models included in (Table 4) had an $\left(\mathrm{R}^{2} \%\right)$ values less than $70 \%$, and multiple correlation coefficient values less than 0.700 , consequently those $X_{i}$ traits studied did not fully explain the total variation in corresponding $Y_{i}$ and those $X_{i}$ may not contain a full appropriate biological entities.

The results indicated that for Giza 88, only yield per feddan $\left(\mathrm{Y}_{1}\right)$ resulted in a model with high $\mathrm{R}^{2}$ value $(99.27 \%$ ), while all other models had unacceptable level of $\mathrm{R}^{2}$ values. The linear regression model for yield per feddan included $\mathrm{X}_{9}$, $\mathrm{X}_{2}, \mathrm{X}_{1}, \mathrm{X}_{7}$ and $\mathrm{X}_{8}$, where two of that $\mathrm{X}_{\mathrm{i}}$ had negative partial regression coefficient $\left(\mathrm{X}_{1}\right.$ and $\left.\mathrm{X}_{8}\right)$. Multiple correlation coefficient value was 0.993 which indeed can be explained that those $X_{i}$ included in the model contain the appropriate biological entities (Table 5).

Data presented in (Tables 6, 7 and 8) followed the same trend discussed in (Table 4). However, the yield per feddan model included $\mathrm{X}_{7}$, and $\mathrm{X}_{2}$ traits only for Giza 80 (Table 6) and Giza 86 (Table 7) and $X_{8}, X_{7}$, and $X_{2}$ traits only for Giza 70 (Table 8). The only exception was in (Table 6), where yield per plant $\left(\mathrm{Y}_{2}\right)$ had $\mathrm{R}^{2}$ value $83.69 \%$ 
Table (3): Average values of yield and yield component traits as affected by microenvironment for 5 cotton varieties (combined data).

\begin{tabular}{|c|c|c|c|c|c|c|}
\hline \multirow[b]{2}{*}{$\begin{array}{l}\text { Microenvironments } \\
\text { (M) }\end{array}$} & \multicolumn{5}{|c|}{ Varieties (V) } & \multirow[b]{2}{*}{ Mean } \\
\hline & $\begin{array}{c}\text { Giza } \\
90 \\
\text { (V1) }\end{array}$ & $\begin{array}{c}\text { Giza } \\
88 \\
\text { (V2) }\end{array}$ & $\begin{array}{c}\text { Giza } \\
88 \\
(V 3)\end{array}$ & $\begin{array}{c}\text { Giza } \\
86 \\
(\mathrm{~V} 4)\end{array}$ & $\begin{array}{c}\text { Giza } \\
\mathbf{7 0} \\
\text { (V5) }\end{array}$ & \\
\hline \multicolumn{7}{|c|}{ Seed cotton (kentar/fed.) } \\
\hline Early time (M1) & 8.86 & 5.57 & 7.81 & 6.69 & 4.49 & 6.68 \\
\hline Moderate time (M2) & 7.61 & 4.31 & 6.60 & 5.45 & 3.24 & 5.44 \\
\hline Late time (M3) & 6.37 & 3.07 & 5.31 & 4.21 & 1.99 & 4.19 \\
\hline Mean & 7.61 & 4.31 & 6.57 & 5.45 & 3.24 & 5.43 \\
\hline LSD 5\% & & $\mathbf{M}=$ & & $\mathrm{V}=\mathbf{0}$ & & \\
\hline \multicolumn{7}{|c|}{ Seed cott } \\
\hline Early time (M1) & 47.72 & 21.20 & 38.65 & 27.81 & 15.26 & 30.12 \\
\hline Moderate time (M2) & 35.88 & 11.96 & 25.76 & 19.29 & 10.29 & 20.63 \\
\hline Late time (M3) & 25.79 & 7.26 & 20.35 & 12.26 & 6.60 & 14.45 \\
\hline Mean & 36.46 & 13.47 & 28.25 & 19.78 & 10.72 & 21.73 \\
\hline LSD 5\% & & $\mathbf{M}=$ & & $\mathrm{V}=3$ & & \\
\hline \multicolumn{7}{|c|}{ per plant } \\
\hline Early time (M1) & 16.07 & 11.09 & 14.48 & 12.52 & 9.36 & 12.70 \\
\hline Moderate time (M2) & 13.35 & 8.24 & 11.86 & 10.22 & 7.47 & 10.22 \\
\hline Late time (M3) & 10.75 & 5.95 & 9.22 & 7.67 & 5.33 & 7.78 \\
\hline Mean & 13.39 & 8.42 & 11.85 & 10.13 & 7.39 & 10.23 \\
\hline LSD 5\% & & & & $\mathbf{V}=1$ & & .24 \\
\hline \multicolumn{7}{|c|}{$\begin{array}{l}\mathrm{M}=1.0 \mathrm{D} \\
\text { Weight of cottor }\end{array}$} \\
\hline Early time (M1) & 3.04 & 2.12 & 2.81 & 2.39 & 1.92 & 2.45 \\
\hline Moderate time M2) & 2.78 & 1.81 & 2.36 & 2.18 & 1.70 & 2.16 \\
\hline Late time (M3) & 2.60 & 1.64 & 2.17 & 1.95 & 1.56 & 1.98 \\
\hline Mean & 2.80 & $\mathbf{1 . 8 5}$ & 2.44 & 2.17 & 1.73 & 2.20 \\
\hline LSD 5\% & & $M=1$ & & $\mathrm{~V}=\mathbf{0}$ & & .21 \\
\hline \multicolumn{7}{|c|}{ ge } \\
\hline Early time (M1) & 40.56 & 36.05 & 39.07 & 37.55 & 35.15 & 37.67 \\
\hline Moderate time (M2) & 37.16 & 34.15 & 36.56 & 35.05 & 32.05 & 34.99 \\
\hline Late time (M3) & 36.56 & 32.16 & 35.06 & 33.56 & 30.56 & 33.58 \\
\hline Mean & 38.09 & 34.12 & 36.89 & 35.38 & 32.59 & 35.41 \\
\hline LSD 5\% & & $\mathbf{M}=$ & & $\mathrm{V}=1$ & & \\
\hline \multicolumn{7}{|c|}{ Seed ind } \\
\hline Early time (M1) & 11.15 & 8.11 & 9.90 & 9.13 & 7.09 & 9.07 \\
\hline Moderate time (M2) & 9.65 & 6.62 & 8.64 & 7.63 & 5.61 & 7.63 \\
\hline Late time (M3) & 8.41 & 5.37 & 7.39 & 6.38 & 4.36 & 6.38 \\
\hline Mean & 9.73 & 6.70 & 8.64 & 7.71 & 5.69 & 7.69 \\
\hline LSD 5\% & & $\mathbf{M}=$ & & $\mathrm{V}=2$ & & \\
\hline \multicolumn{7}{|c|}{ Lint ind } \\
\hline Early time (M1) & 7.44 & 4.51 & 6.36 & 5.39 & 3.71 & 5.48 \\
\hline Moderate time (M2) & 5.82 & 3.31 & 4.88 & 4.06 & 2.63 & 4.14 \\
\hline Late time (M3) & 4.76 & 2.53 & 3.93 & 3.19 & 1.93 & 3.26 \\
\hline Mean & 6.00 & 3.45 & 5.05 & 4.21 & 2.76 & 4.29 \\
\hline LSD 5\% & \multicolumn{5}{|c|}{$M=1.41$} & \\
\hline
\end{tabular}


Table (4): Linear regression models, coefficients of determination $\left(\mathbf{R}^{2}\right)$ and multiple correlation coefficients $(R)$ for Giza 90 variety based on data combined over years and micro-environments.

\begin{tabular}{|c|c|c|c|}
\hline Variables & Linear Regression Models & $\mathbf{R}^{2} \%$ & $\mathbf{R}$ \\
\hline Yield / feddan $\left(\mathrm{Y}_{1}\right)$ & $\begin{array}{c}\hat{Y}_{1}=-9.48+0.18 X_{7}+0.12 X_{2}- \\
0.05 X_{1}\end{array}$ & 99.05 & 0.991 \\
\hline Yield / plant $\left(Y_{2}\right)$ & $\begin{array}{c}\hat{Y}_{2}=-129.54+2.05 X_{2}+5.47 X_{9}- \\
3.50 X_{1}+1.11 X_{7}\end{array}$ & 63.35 & 0.634 \\
\hline $\begin{array}{l}\text { No. of open bolls / } \\
\text { plant }\left(\mathbf{Y}_{3}\right)\end{array}$ & $\begin{array}{c}\hat{Y}_{3}=-55.92+1.03 X_{7}+1.02 X_{3}- \\
0.31 X_{8}+0.28 X_{4}\end{array}$ & 82.73 & 0.827 \\
\hline Boll weight $\left(\mathbf{Y}_{4}\right)$ & $\begin{array}{c}\hat{\mathbf{Y}}_{4}=0.681+0.36 X_{2}+0.14 X_{9}+ \\
0.24 X_{7}-0.03 X_{6}\end{array}$ & $\mathbf{5 9 . 0 7}$ & 0.591 \\
\hline $\begin{array}{l}\text { Lint percentage } \\
\left(Y_{5}\right)\end{array}$ & $\hat{Y}_{5}=11.49+0.37 X_{7}-0.73 X_{6}$ & 49.25 & 0.493 \\
\hline Seed index $\left(Y_{6}\right)$ & $\begin{array}{c}\hat{Y}_{6}=-9.62+0.21 X_{7}-0.36 X_{1}+ \\
0.61 X_{9}+0.09 X_{2}\end{array}$ & 69.57 & 0.696 \\
\hline Lint index $\left(\mathrm{Y}_{7}\right)$ & $\begin{array}{c}\hat{Y}_{7}=-27.10+0.24 X_{7}-0.36 X_{4}+ \\
0.73 X_{10}\end{array}$ & 55.79 & 0.558 \\
\hline
\end{tabular}

Table (5): Linear regression models, coefficients of determination $\left(\mathbf{R}^{2}\right)$ and multiple correlation coefficients $(R)$ for Giza 88 variety based on data combined over years and micro-environments.

\begin{tabular}{|c|c|c|c|}
\hline Variables & Linear Regression Models & $\mathbf{R}^{2} \%$ & $\mathbf{R}$ \\
\hline $\begin{array}{l}\text { Yield / feddan } \\
\left(Y_{1}\right)\end{array}$ & $\begin{array}{l}\hat{Y}_{1}=-129.54+5.47 X_{9}+2.05 X_{2} \\
-3.50 X_{1}+1.11 X_{7}=0.09 X_{8}\end{array}$ & 99.27 & 0.993 \\
\hline Yield / plant $\left(\mathbf{Y}_{2}\right)$ & $\hat{Y}_{2}=-30.60+0.87 X_{2}-0.33 X_{1}$ & 59.09 & 0.591 \\
\hline $\begin{array}{l}\text { No. of open bolls / } \\
\text { plant }\left(\mathbf{Y}_{3}\right)\end{array}$ & $\begin{array}{l}\hat{Y}_{3}=-81.15+1.32 X_{7}+2.37 X_{3}+ \\
1.95 X_{9}+1.52 X_{5}\end{array}$ & 73.53 & 0.735 \\
\hline Boll weight $\left(\mathbf{Y}_{4}\right)$ & $\hat{\mathbf{Y}}_{4}=-4.73+0.04 X_{7}+0.03 X_{5}$ & $\mathbf{5 7 . 5 3}$ & 0.575 \\
\hline $\begin{array}{c}\text { Lint percentage } \\
\left(Y_{5}\right)\end{array}$ & $\hat{Y}_{5}=5.34+0.64 X_{2}+0.53 X_{10}$ & 41.79 & 0.418 \\
\hline Seed index $\left(Y_{6}\right)$ & $\begin{array}{l}\hat{Y}_{6}=-11.92+0.28 X_{10}+0.11 X_{7}- \\
0.44 X_{9}\end{array}$ & 55.64 & 0.556 \\
\hline Lint index $\left(\mathrm{Y}_{7}\right)$ & $\begin{array}{l}\hat{Y}_{7}=-11.18+0.13 X_{7}+.015 X_{2}- \\
0.16 X_{9}\end{array}$ & 47.17 & 0.472 \\
\hline
\end{tabular}

Table (6):Linear regression models, coefficients of determination $\left(R^{2}\right)$ and multiple correlation coefficients $(R)$ for Giza 80 variety based on data combined over years and micro-environments.

\begin{tabular}{|l|c|c|c|}
\hline Variables & Linear Regression Models & $R^{2} \%$ & $R$ \\
\hline Yield / feddan $\left(Y_{1}\right)$ & $\hat{Y}_{1}=-10.21+0.41 X_{7}+0.29 X_{2}$ & 99.51 & 0.995 \\
\hline Yield / plant $\left(Y_{2}\right)$ & $\begin{array}{c}\hat{Y}_{2}=-791.18+5.24 X_{8}+1.60 X_{7^{-}} \\
0.80 X_{2}\end{array}$ & 83.69 & 0.837 \\
\hline $\begin{array}{l}\text { No. of open bolls / } \\
\text { plant }\left(Y_{3}\right)\end{array}$ & $\begin{array}{c}\hat{Y}_{3}=-52.67+0.36 X_{3}+0.51 X_{2}+ \\
0.17 X_{7}+0.94 X_{9}\end{array}$ & 47.45 & 0.475 \\
\hline Boll weight $\left(Y_{4}\right)$ & $\begin{array}{c}\hat{Y}_{4}=-7.38+0.06 X_{7}+0.02 X_{1}+ \\
0.05 X_{5}-0.36 X_{2}\end{array}$ & 63.21 & 0.632 \\
\hline Lint percentage $\left(Y_{5}\right)$ & $\begin{array}{c}\hat{Y}_{5}=6.80+0.31 X_{7}+0.22 X_{2}+0.17 \\
X_{10}\end{array}$ & 56.59 & 0.566 \\
\hline Seed index $\left(Y_{6}\right)$ & $\begin{array}{c}\hat{Y}_{6}=-21.82+0.13 X_{7}+0.10 X_{2^{-}} \\
0.22 X_{1}+0.15 X_{5}\end{array}$ & 62.12 & 0.621 \\
\hline Lint index $\left(Y_{7}\right)$ & $\hat{Y}_{7}=-13.06+0.18 X_{7}+0.15 X_{2}$ & 67.05 & 0.671 \\
\hline
\end{tabular}


Table (7): Linear regression models, coefficients of determination $\left(\mathbf{R}^{2}\right)$ and multiple correlation coefficients $(R)$ for Giza 86 variety based on data combined over years and microenvironments.

\begin{tabular}{|l|c|c|c|}
\hline Variables & Linear Regression Models & $R^{2} \%$ & $R$ \\
\hline Yield / feddan $\left(Y_{1}\right)$ & $\hat{Y}_{1}=10.18+0.35 X_{7}+0.33 X_{2}$ & 99.41 & 0.994 \\
\hline Yield / plant $\left(Y_{2}\right)$ & $\begin{array}{c}\hat{Y}_{2}=-180.52+1.60 X_{2}-0.47 X_{1}+0.60 X_{5}+ \\
0.42 X_{7}\end{array}$ & 54.39 & 0.544 \\
\hline $\begin{array}{c}\text { No. of open bolls / plant } \\
\left(Y_{3}\right)\end{array}$ & $\hat{Y}_{3}=-\mathbf{5 3 . 5 0}+0.59 X_{2}+0.29 X_{5}+0.58 X_{4}$ & 57.33 & 0.573 \\
\hline Boll weight $\left(Y_{4}\right)$ & $\begin{array}{c}\hat{Y}_{4}=-3.33+0.07 X_{2}+0.06 X_{6}-0.12 X_{1}+ \\
0.15 X_{10}\end{array}$ & 46.15 & 0.462 \\
\hline Lint percentage $\left(Y_{5}\right)$ & $\hat{Y}_{5}=-12.60+0.30 X_{7}+0.21 X_{5}$ & 65.47 & 0.655 \\
\hline Seed index $\left(Y_{6}\right)$ & $\hat{Y}_{6}=-\mathbf{0 . 0 9} 0.17 X_{7}+0.39 X_{5}-0.29 X_{8}$ & 54.21 & 0.542 \\
\hline Lint index $\left(Y_{7}\right)$ & $\hat{Y}_{7}=-21.51+0.13 X_{7}+0.16 X_{5}-0.04 X_{1}-$ & 69.48 & 0.695 \\
& $0.09 X_{4}$ & & \\
\hline
\end{tabular}

Table (8): Linear regression models, coefficients of determination $\left(R^{2}\right)$ and multiple correlation coefficients $(R)$ for Giza 70 variety based on data combined over years and micro-environments.

\begin{tabular}{|l|l|c|c|}
\hline Variables & Linear Regression Models & $R^{2} \%$ & $R$ \\
\hline Yield / feddan $\left(Y_{1}\right)$ & $\hat{Y}_{1}=-149.85+0.94 X_{8}+0.42 X_{7}+0.28 X_{2}$ & 99.37 & 0.994 \\
\hline Yield / plant $\left(Y_{2}\right)$ & $\hat{Y}_{2}=-13.78+1.80 X_{2}-0.66 X_{1}-1.21 X_{6}$ & 33.25 & 0.333 \\
\hline No. of open bolls / plant $\left(Y_{3}\right)$ & $\hat{Y}_{3}=-31.56+0.85 X_{2}+0.57 X_{10}$ & 84.79 & 0.848 \\
& $\hat{Y}_{4}=-3.50+0.03 X_{7}+0.03 X_{3}$ & 58.09 & 0.581 \\
\hline Boll weight $\left(Y_{4}\right)$ & $\hat{Y}_{5}=0.70+0.72 X_{2}-2.17 X_{9}$ & 63.22 & 0.632 \\
\hline Lint percentage $\left(Y_{5}\right)$ & $\hat{Y}_{6}=-120.56+0.41 X_{7}+0.84 X_{8}-0.18 X_{5}$ & 59.13 & 0.591 \\
\hline Seed index $\left(Y_{6}\right)$ & $\hat{Y}_{7}=\mathbf{2 0 . 4 4}+0.16 X_{7}+0.12 X_{3}-0.04 X_{1}-$ & 43.17 & 0.432 \\
\hline Lint index $\left(Y_{7}\right)$ & $0.04 X_{2}$ & & \\
\hline
\end{tabular}

where the model included $\mathrm{X}_{8}, \mathrm{X}_{7}$ and $\mathrm{X}_{2}$ traits.

It could be concluded that earliness traits were able to result in a linear regression model for all the five tested entries and had basic biological entities.

\section{REFERENCES}

Abdel-Rahman L. M. A. (1983). Studies on fiber and seed development of cotton. Ph. D. Thesis, Fac. of Agric., Cairo University, Egypt.

Badr S. S. M., Hassan I. S. M. and Laila AbdelRahman M. A. (2001). Comparative study on flowering, yield components and lint properties of some new Egyptian cotton cultivars. J. Agric. Sci. Mansoura, 26 (6): 3473-3486.

Bartlett M. S. (1973). Some examples of statistical methods of research in agriculture and applied botany. In Roy Statist, Soc., B., 4:37-79.

Bliboro J. D. and Quisenberry J. E. (1973). A yield related measure of earliness for cotton. (Gossypium hirsutum L.). Crop Sci., 13: 392 - 393.

Christidis B. G. and Harrison G. J. (1955). Cotton growing problems. McGraw-Hill Book Co., Inc., New York, PP. 633.

Cochran W. G. and Cox G.W. (1957). Experimental design (2nd Ed.). John Wiley and Sons, Inc,. New York.

Draper N. R. and Smith H. (1966). Applied regression analysis. John Wiley and Sons. Inc, New York - 407 PP.

El-Beily M. A., Abou-Tour H. B., and Okasha A. A. (1996). The relative contribution of yield components to lint yield in three cultivars of Egyptian cotton. J. Agric. Res. Tanta Univ., 22 (1):61-67.

El-Shaer M.H., Shabana R., El-Shiekh A. H. and Abdel-Rahman L. M. A. (1984). Path coefficient analysis of some characters contribution to yield and quality of cotton. Agric. Res. Rev. Vol., 62 No (6): 23-31.

Gopang A.D. (2003). Studies on correlation of some morphological characters with 
earliness in cotton. Sci. Technol. Devel., 22(3):23- 31 .

Hassan I. S. M. and Abdel-Aziz A. M. (2004). Multivariate analysis of yield and relative contribution of variables to variation in cotton yield. J. Agric. Sci. Mansoura Univ., 29 (10): 5467 - 5473

Ismail, F.M. and Al-Enani F.A. (1986). Comparative study for the relative contribution to seed cotton yields in American and Egyptian cottons. Crop Sci., 156: $128-132$.

Karademir C., Karademir E., Ekinci R. and Gencer O. (2009). Correlations and path coefficient analysis between leaf chlorophyll content, yield and yield components in cotton (Gossypium hirsutum L.) under drought stress conditions. Not. Bot. Hort. Agrobot. Cluj., 37 (2): 241-24.

Kausar M. N. S., Malik S. A. and Saleem M. (2005). Stability of cotton cultivars for early crop maturity across variable plant spacing and sowing times. Pak. J. Bot., 37(2): 345 353.

Kazerani B. (2012). Determination of the best cotton cultivars and selection criteria to improve yield in Gorgan climatic region. Afr. J. Agric. Res., 7(11): 1719 - 1726.

Muhammad I., Chang M. A., Iqbal M. Z., Hassan M. and Noor-El-Islam A. (2003). Correlation and path coefficient analysis of earliness and agronomic characters of upland cotton in Multan. Pak. J. of Agro., 2(3):160-168.
Muhammad I., Chang M. A., Iqbal M. Z., Hassan M.and Noor-El-Islam A. (2006). Correlation and path coefficient analysis for earliness and yield traits in cotton $(G$. hirsutum). Asian J. of Plant Sci., 5: (2): 341-344.

Ray T. R. and Richmond T. R. (1966). Morphological measures of earliness of crop maturity in cotton. Crop Sci., 6: 527531.

Richmond T. R. and Ray T. R. (1966). Product quantity measures of earliness of crop maturity in cotton. Crop Sci., 6: 235 - 239.

Saeed A., Ahmad S., Ashraf M., Islamkham N. and Iqbals N. (2008) Assessment of yieldrelated morphological measures for earliness in Upland cotton (Gossypium hirsutum L.). Pak. J. Bot., 40 (3):12011207.

Seyam S. M., Abo El-Zahab A. A., El-Rassas H. N. and El-Rayse F. M. (1984). The use of stepwise regression analysis in determining characters for yield selection in Egyptian cotton. Agric. Res. Rev., 62 (6):41-47.

Shazia S. A., Rehman A. and Iqbal K. (2010). Correlations analysis of seed cotton yields with some quantitative traits in Upland cotton (Gossypium hirsutum L.). Pak. J. Bot., 42(6): 3799 - 3805.

Snedecor G. A. and Cochran A. D. (1981). Statistical methods $7^{\text {th }}$ Edition, Iowa State Univ.

Young E. F., R. M. Taylor J.Y. and Peterse H. D. (1980). Days dgree unit and time in relation to vegetative development and fruiting for three cultivars of cotton. Crop Sci., 20: 320 -375 .

\footnotetext{
تحليل الإحدار والإرتباط المتعدد لصفات التبكير والقدرة الصنفية 


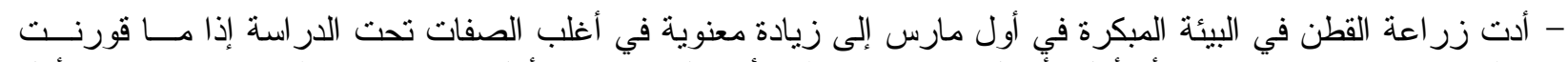

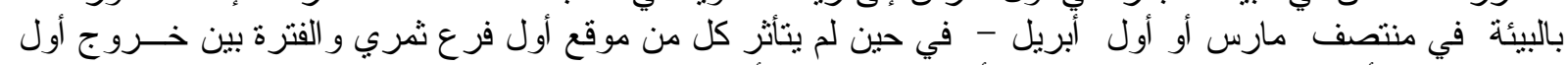

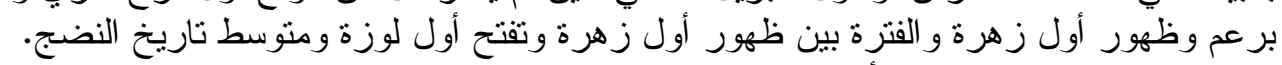

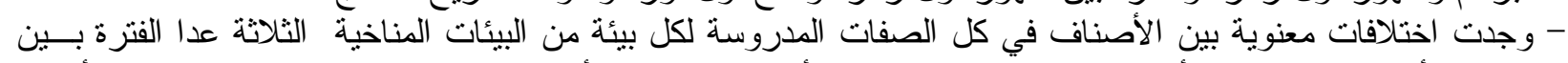

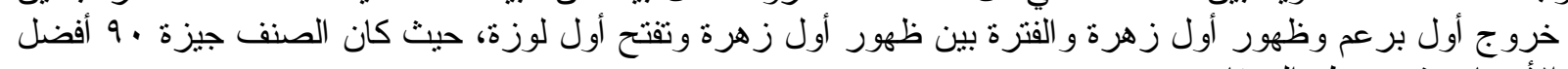
الأصناف في معظم الصفات.

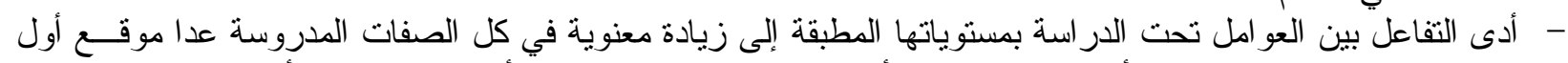

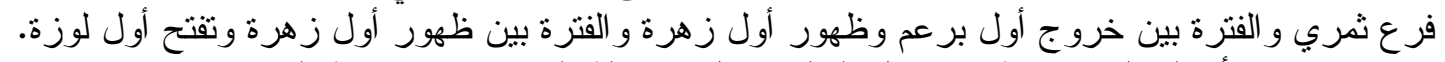

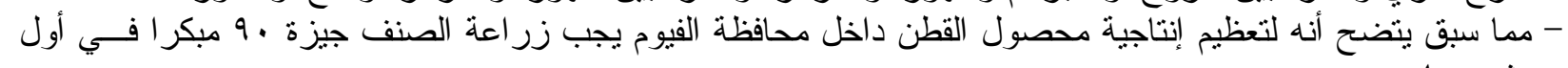
- يمكن من النتائج الخاصة بمعاملات الإنحدار الخطي المتعدد استتناج أن متغير ات التبكير قد أثرت بصورة أو بأخري في

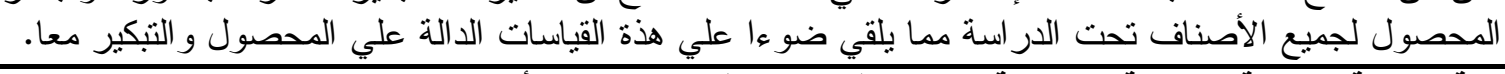

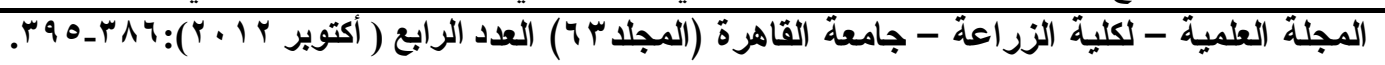

